\title{
EXTENSIONS OF ABSTRACT VALUED SET FUNCTIONS
}

\author{
BY \\ JAMES E. HUNEYCUTT, JR.(1)
}

\section{CHAPTER I}

In measure theory, an essential concept is the extension of the notion of a "measure" on one class of sets to a notion of measure on a larger class of sets. This paper investigates such extensions when the "measure" takes values in a topological abelian group. We also investigate relationships between regularity and countable additivity.

It is shown that under sufficiently strong regularity conditions, a modular function on a lattice determines a unique countably additive function on the semiring generated by the lattice. Similar theorems are derived showing that finitely additive functions which are sufficiently regular on a ring or semiring are also countably additive. The final chapter investigates the extension of a Banach space valued, modular function on a lattice to a countably additive function on the $\sigma$-ring generated by the lattice.

\section{Chapter II}

Let $X$ be a set and $\mathscr{H}$ a semiring of subsets of $X$; let $\mathscr{G}$ be an abelian group. A function $\mu: \mathscr{H} \rightarrow \mathscr{G}$ is additive provided that for each finite, pairwise disjoint sequence $\left\{A_{i}\right\}_{1}^{N}$ in $\mathscr{H}$ whose union is in $\mathscr{H}, \mu\left(\bigcup_{1}^{N} A_{i}\right)=\sum_{1}^{N} \mu\left(A_{i}\right)$. Von Neumann $[5$, p. 94] showed that any additive function on a semiring has a unique additive extension to the smallest ring $\mathscr{R}(\mathscr{H})$ containing $\mathscr{H}$. If $\mathscr{G}$ is a topological abelian group, we can define countable additivity of an additive function $\mu: \mathscr{H} \rightarrow \mathscr{G}$ by requiring that $\left\{\sum_{1}^{N} \mu\left(A_{i}\right)\right\}_{N=1}^{\infty}$ converges to $\mu\left(\bigcup_{1}^{\infty} A_{i}\right)$ whenever $\left\{A_{i}\right\}_{1}^{\infty}$ is a pairwise disjoint sequence of members of $\mathscr{H}$ whose union is a member of $\mathscr{H}$. Von Neumann also showed that if the function $\mu: \mathscr{H} \rightarrow \mathscr{G}$ is countably additive, then the unique additive extension to the ring $\mathscr{R}(\mathscr{H})$ is also countably additive. Thus it is of interest to know conditions under which an additive function on a semiring to a topological abelian group will be countably additive. One such condition ensuring countable additivity is regularity.

By saying that $\mu: \mathscr{H} \rightarrow \mathscr{G}$ is regular, we usually mean that each member of $\mathscr{H}$ can be "approximated from below by compact sets" and "approximated from above by open sets."

Presented to the Society, January 26, 1968 under the title Regular additive set functions and January 25, 1969 under the title Extensions of vector-valued modular functions; received by the editors September 13, 1968.

(1) The results presented in this paper are a part of the author's Ph.D. dissertation, written at the University of North Carolina at Chapel Hill under the direction of Professor B. J. Pettis. 
Suppose $X$ is a topological space and $\mathscr{H}$ is a semiring of subsets of $X$. In case $\mu$ is a nonnegative, additive function on $\mathscr{H}$, we follow Von Neumann by defining regularity as follows:

$R_{V N}:$ For each $A$ in $\mathscr{H}$ and $\varepsilon>0$, there exist $C$ compact, $U$ open, $A^{\prime}, A^{\prime \prime} \in \mathscr{H}$ such that $A^{\prime} \subseteq C \subseteq A \subseteq U \subseteq A^{\prime \prime}$ and $\mu\left(A^{\prime \prime}\right)-\mu\left(A^{\prime}\right)<\varepsilon$.

Von Neumann showed that if a nonnegative, additive function on $\mathscr{H}$ satisfies $R_{V N}$, then the function is countably additive. When the function takes values in a set with less structure than the nonnegative reals (e.g. the complex numbers, a linear topological space, or a topological abelian group) then there are various ways in which regularity can be defined. We seek one sufficiently strong to extend finite additivity to countable additivity but sufficiently weak to be useful. We shall also require that it reduce to $R_{V N}$ in the case in which the function takes only nonnegative real values.

The topology for a topological abelian group $\mathscr{G}$ is generated by a family $\left\{\|\cdot\|_{p}: p \in P\right\}$ of seminorms. For each $p$ in $P$ and each subset $B$ of $X$ we define

(i) $\left(\mu_{R}\right)_{p}(B)=\sup \left\{\|\mu(A)\|_{p}: A \in \mathscr{G}, A \subseteq B\right\}$,

(ii) $\left(\mu_{D}\right)_{p}(B)=\sup \left\{\left\|\sum_{1}^{N} \mu\left(A_{i}\right)\right\|_{p}\right\}$,

(iii) $|\mu|_{p}(B)=\sup \left\{\sum_{1}^{N}\left\|\mu\left(A_{i}\right)\right\|_{p}\right\}$,

where the supremum in (ii) and (iii) is taken over all finite, pairwise-disjoint sequences in $\mathscr{H}$ whose union is a subset of $B$.

In the statement of $R_{V N}$, we require that " $\mu\left(A^{\prime \prime}\right)-\mu\left(A^{\prime}\right)<\varepsilon$ ". If $\hat{\mu}$ is the unique additive extension of $\mu$ to $\mathscr{R}(\mathscr{H})$, then this requirement is equivalent to " $\hat{\mu}\left(A^{\prime \prime} \backslash A^{\prime}\right)$ $<\varepsilon$ "; and thus the requirement is that $A^{\prime \prime} \backslash A^{\prime}$ be small in some sense. The preceding observation motivates the following

Definition. $\mu$ is $\mu_{R}$-regular on $\mathscr{H}$ provided that for each $A$ in $\mathscr{H}, p \in P$ and $\varepsilon>0$, there exist $C$ countably compact, $U$ open, $A^{\prime}, A^{\prime \prime} \in \mathscr{H}$ such that $A^{\prime} \subseteq C \subseteq A \subseteq U \subseteq A^{\prime \prime}$ and $\left(\mu_{R}\right)_{p}\left(A^{\prime \prime} \backslash A^{\prime}\right)<\varepsilon$. Similar definitions are made for $\mu_{D}$-regularity and $|\mu|-$ regularity.

We note that if $\mu$ takes only nonnegative real values, then $|\mu|\left(A^{\prime \prime} \backslash A^{\prime}\right)=\mu_{D}\left(A^{\prime \prime} \backslash A^{\prime}\right)$ $=\mu_{R}\left(A^{\prime \prime} \mid A^{\prime}\right)=\mu\left(A^{\prime \prime}\right)-\mu\left(A^{\prime}\right)$ and each regularity becomes $R_{V N}$.

Let $\mathscr{C}$ denote the collection of countably compact sets; and $\mathscr{T}$, the collection of open sets.

THEOREM 2.1. If $\mu$ is finitely additive and $\mu_{D}$-regular, then $\mu$ is countably additive.

Proof. Let $\left\{B_{n}\right\}^{\infty}$, be a pairwise-disjoint sequence of members of $\mathscr{H}$ such that $\cup_{1}^{\infty} B_{n}=B \in \mathscr{H}$. Let $\varepsilon>0$ and $p \in P$. Then $\exists F, G \in \mathscr{H}, C \in \mathscr{C}, U \in \mathscr{T}$ such that $F \subseteq C \subseteq B \subseteq U \subseteq G$ and $\left(\mu_{D}\right)_{p}(G \mid F)<\varepsilon$. Similarly, for each $n, \exists F_{n}, G_{n} \in \mathscr{H}, C_{n} \in \mathscr{C}$, and $U_{n} \in \mathscr{T}$ such that $F_{n} \subseteq C_{n} \subseteq B_{n} \subseteq U_{n} \subseteq G_{n}$ and $\left(\mu_{D}\right)_{p}\left(G_{n} \mid F_{n}\right)<\varepsilon / 2^{n}$. 
(i) For each positive integer $N,\left(\mu_{D}\right)_{p}\left[\left(\bigcup_{1}^{N} G_{n}\right) \mid F\right]<2 \varepsilon$ :

$$
\begin{aligned}
\left(\bigcup_{1}^{N} G_{n}\right) \backslash F & \subseteq\left[\left(\bigcup_{1}^{N} G_{n}\right) \backslash B\right] \cup[B \backslash F] \\
& =\left[\left(\bigcup_{1}^{N} G_{n}\right) \backslash \bigcup_{1}^{\infty} B_{n}\right] \cup[B \backslash F] \subseteq\left[\bigcup_{1}^{N}\left(G_{n} \backslash B_{n}\right)\right] \cup[B \backslash F] .
\end{aligned}
$$

It is easily seen that $\left(\mu_{D}\right)_{p}$ is monotone and subadditive on $\mathscr{R}(\mathscr{H})$, so

$$
\left(\mu_{D}\right)_{p}\left[\left(\bigcup_{1}^{N} G_{n}\right) \backslash F\right] \leqq \sum_{1}^{N}\left(\mu_{D}\right)_{p}\left(G_{n} \mid B_{n}\right)+\left(\mu_{D}\right)_{p}(B \backslash F)<\sum_{1}^{N} \varepsilon / 2^{n}+\varepsilon<2 \varepsilon .
$$

(ii) $\|\mu(B)-\mu(F)\|_{p}<\varepsilon$ : Let $\hat{\mu}$ be the unique finitely additive extension of $\mu$, defined on the smallest ring containing $\mathscr{H}$. Then

$$
\|\mu(B)-\mu(F)\|_{p}=\|\hat{\mu}(B \mid F)\| \leqq\left(\hat{\mu}_{R}\right)_{p}(B \mid F)=\left(\mu_{D}\right)_{p}(B \mid F)<\varepsilon .
$$

(iii) There is a positive integer $M$ such that for any positive integer $N \geqq M$, we have $\left\|\mu(F)-\sum_{1}^{N} \mu\left(B_{n}\right)\right\|_{p}<3 \varepsilon: F \subseteq C \subseteq B \subseteq \bigcup_{1}^{\infty} B_{n} \subseteq \bigcup_{1}^{\infty} U_{n}$ and $C$ is countably compact, so there is a positive integer $M$ such that $F \subseteq C \subseteq \bigcup_{1}^{M} U_{n} \subseteq \bigcup_{1}^{M} G_{n}$. For $N \geqq M$ we have

$$
\begin{aligned}
\left\|\mu(F)-\sum_{1}^{N} \mu\left(B_{n}\right)\right\|_{p} & \leqq\left\|\hat{\mu}\left(\bigcup_{1}^{N} B_{n}\right)-\hat{\mu}\left(\bigcup_{1}^{N} G_{n}\right)\right\|_{p}+\left\|\hat{\mu}\left(\bigcup_{1}^{N} G_{n}\right)-\hat{\mu}(F)\right\|_{p} \\
& =\left\|\hat{\mu}\left[\left(\bigcup_{1}^{N} G_{n}\right) \backslash\left(\bigcup_{1}^{N} B_{n}\right)\right]\right\|_{p}+\left\|\hat{\mu}\left[\left(\bigcup_{1}^{N} G_{n}\right) \backslash F\right]\right\|_{p} \\
& \leqq\left(\mu_{D}\right)_{p}\left[\left(\bigcup_{1}^{N} G_{n}\right) \backslash\left(\bigcup_{1}^{N} B_{n}\right)\right]+\left(\mu_{D}\right)_{p}\left[\left(\bigcup_{1}^{N} G_{n}\right) \backslash F\right] \\
& <\left(\mu_{D}\right)_{p}\left[\bigcup_{1}^{N}\left(G_{n} \backslash B_{n}\right)\right]+2 \varepsilon \leqq \sum_{1}^{N}\left(\mu_{D}\right)_{p}\left(G_{n} \mid B_{n}\right)+2 \varepsilon<3 \varepsilon .
\end{aligned}
$$

(iv) For the $M$ derived in (iii), $\left\|\mu(B)-\sum_{1}^{N} \mu\left(B_{n}\right)\right\|_{p}<4 \varepsilon$ whenever $N \geqq M$ :

$$
\left\|\mu(B)-\sum_{1}^{N} \mu\left(B_{n}\right)\right\|_{p} \leqq\|\mu(B)-\mu(F)\|_{p}+\left\|\mu(F)-\sum_{1}^{N} \mu\left(B_{n}\right)\right\|_{p}<\varepsilon+3 \varepsilon=4 \varepsilon \text {. }
$$

Thus $\sum_{1}^{N} \mu\left(B_{n}\right) \rightarrow \mu(B)$.

From the remark immediately preceding the theorem, we see that when $\mu$ takes only nonnegative real values, Von Neumann's result is obtained as a corollary. We also have the following immediate corollaries

COROLlARY. If $\mathscr{R}$ is a ring of sets and $\mu: \mathscr{R} \rightarrow \mathscr{G}$ is finitely additive and $\mu_{R}$-regular, then $\mu$ is countably additive.

COROLlaRY. If $\mathscr{H}$ is a semiring of sets and $\mu: \mathscr{H} \rightarrow \mathscr{G}$ is finitely additive and $|\mu|-$ regular, then $\mu$ is countably additive.

Dinculeanu and Kluvanek [2] have recently published the result contained in the first corollary for the special case in which $\mathscr{G}$ is a locally convex linear topological space. 
Since $\mu_{R}$-regularity and finite additivity for a ring are sufficient to ensure countable additivity, it is of interest to ask whether $\mu_{R}$-regularity for a semiring is sufficient to produce countable additivity from finite additivity. This question can be answered in the negative by the following

EXAMPLE. Let $\mathscr{H}$ be the semiring of all real intervals of the form $(a, b]$ where $a \leqq b$. Let

$$
\begin{array}{cl}
F(x)=x \sin \pi / x & \text { if } x \neq 0 \\
=0 & \text { if } x=0 .
\end{array}
$$

Then $\mu: \mathscr{H} \rightarrow R$ by $\mu(a, b]=F(b)-F(a)$ is finitely additive. Moreover $\mu$ is $\mu_{R^{-}}$ regular but not $\mu_{D}$-regular. It can be shown by using the fact that $F$ is not of bounded variation on $[-1,1]$ that $\mu$ is not countably additive. A subsequent paper will treat the relations between regularity and functions of bounded variation.

We note that the results of this chapter can be divorced from a topological space setting by hypothesizing the existence of two collections $\mathscr{C}$ and $\mathscr{T}$ of subsets of $X$ such that members of $\mathscr{C}$ are countably compact with respect to coverings by members of $\mathscr{T}$. Regularity is then phrased in terms of approximation from below by members of $\mathscr{C}$ and from above by members of $\mathscr{T}$.

\section{CHAPTER III}

Pettis [7, p. 187] has shown that any modular, abelian group valued function $\mu$ on a lattice $\mathscr{L}$ of subsets of a set $X$ determines a unique finitely additive function $\hat{\mu}$ on the semiring $\mathscr{H}(\mathscr{L})$ generated by $\mathscr{L}$ (and this, by Von Neumann's result, can be uniquely extended to the ring generated by $\mathscr{L}$ ). He also defined a type of regularity for a nondecreasing modular function $\mu$ which ensures that the unique (nonnegative) additive function $\hat{\mu}$ be countably additive. In the present chapter, we extend this result to the case in which $\mu$ is topological abelian group valued.

We follow the pattern of Pettis' work and define, for $\mathscr{U}, \mathscr{V} \in 2^{x}$,

$\mathscr{U} \mid \mathscr{V}=\{U \backslash V: U \in \mathscr{U}, V \in \mathscr{V}\}$,

$\mathscr{U} \backslash \mid \mathscr{V}=\{U \backslash V: U \in \mathscr{U}, V \in \mathscr{V}$ and $V \subseteq U\}$,

$\mathscr{C}(\mathscr{U})=\{A \subseteq X: A$ is countably compact with respect to covering by members of $\mathscr{U}\}$.

To obtain a concept of approximation, we make the following

Definition. Let $\mathscr{L}$ be a lattice of subsets of a set $X ; \mathscr{G}$, a topological abelian group whose topology is determined by $\left\{\|\cdot\|_{p}: p \in P\right\}$; and let $\mu: \mathscr{L} \rightarrow \mathscr{G}$ be modular. For $B \subseteq X$ and $p \in P$, we define $\left(\mu_{L}\right)_{p}(B)=\sup \left\{\left\|\sum_{1}^{N} \mu\left(A_{i}\right)-\mu\left(C_{i}\right)\right\|_{p}\right\}$ where the supremum is taken over all pairs of finite sequences $\left\{A_{i}\right\}_{1}^{n},\left\{C_{i}\right\}_{1}^{n}$ of members of $\mathscr{L}$ where $C_{i} \subseteq A_{i}(1 \leqq i \leqq n)$ and $\left\{A_{i} \mid C_{i}\right\}_{1}^{n}$ is pairwise disjoint and $\bigcup_{1}^{n}\left(A_{i} \mid C_{i}\right) \subseteq B$.

We note that $\left(\mu_{L}\right)_{p}(B)=\left(\hat{\mu}_{D}\right)_{p}(B)$ and in case $\mu$ is nondecreasing real-valued, $\left(\mu_{L}\right)(A \backslash C)=\mu(A)-\mu(C)$ for $A$ and $C \in \mathscr{L}$ with $C \subseteq A$. We also define $\mathscr{L}(\mathscr{U}, \mathscr{V})$ (for $\mathscr{U}, \mathscr{V} \subseteq 2^{\mathrm{X}}$ ) to be those members $A$ of $\mathscr{L}$ such that for each $\varepsilon>0$ and each $p \in P$, there exist $A^{\prime}, A^{\prime \prime}$ in $\mathscr{L}, U \in \mathscr{U}$, and $V \in \mathscr{V}$ with $A^{\prime} \subseteq U \subseteq A \subseteq V \subseteq A^{\prime \prime}$ and $\left(\mu_{L}\right)_{p}\left(A^{\prime \prime} \backslash A^{\prime}\right)$ 
$<\varepsilon$. Pettis made a similar definition for nondecreasing functions, requiring $\mu\left(A^{\prime \prime}\right)$ $-\mu\left(A^{\prime}\right)<\varepsilon$. By the remark immediately preceding the definition, we see that the present concept of $\mathscr{L}(\mathscr{U}, \mathscr{V})$ does extend his definition.

We have the following generalization of his result (for the original result, see Corollary 3.1.1 below):

THEOREM 3.1. Let $\mathscr{L}$ be a lattice of subsets of a set $X$ and $\mu$ be a function defined on $\mathscr{L}$ with values in the topological abelian group $\mathscr{G}$. Suppose that there exist collections $\mathscr{U}$ and $\mathscr{V}$ of subsets of $X$ such that

(i) $\mathscr{U} \backslash \mathscr{V} \subseteq \mathscr{C}(\mathscr{V} \| \mathscr{U})$,

(ii) $\mathscr{H}(\mathscr{L}) \subseteq \mathscr{L}(\mathscr{U}, \mathscr{V}) \| \mathscr{L}(\mathscr{U}, \mathscr{V})$.

If $\mu$ is modular, then the uniquely determined additive function $\hat{\mu}: \mathscr{H}(\mathscr{L}) \rightarrow \mathscr{G}$ is countably additive (and hence can be uniquely extended to a countably additive function on the ring $\mathscr{R}(\mathscr{H}(\mathscr{L}))$ generated by $\mathscr{L})$.

Proof. By the observation at the end of Chapter II, it is sufficient to find two collections $\mathscr{A}$ and $\mathscr{B}$ of subsets of $X$ such that members of $\mathscr{B}$ are countably compact with respect to coverings by members of $\mathscr{A}$ and $\hat{\mu}$ is regular with respect to approximation from within by members of $\mathscr{B}$ and from without by members of $\mathscr{A}$. Let $\mathscr{A}=\mathscr{U} \backslash \mathscr{V}$ and $\mathscr{B}=\mathscr{V} \| \mathscr{U}$; then by condition (i), members of $\mathscr{B}$ are countably compact with respect to coverings by members of $\mathscr{A}$. Let $A \in \mathscr{H}(\mathscr{L}) \varepsilon>0$, and $p \in P$ $\left(\left\{\|\cdot\|_{p}: p \in P\right\}\right.$ generates the topology of $\left.\mathscr{G}\right)$; then there exist by (ii), $B_{1}$ and $B_{2}$ in $\mathscr{L}(\mathscr{U}, \mathscr{V})$ such that $A=B_{1} \backslash B_{2}$ and $B_{2} \subseteq B_{1}$. Since $B_{1}$ and $B_{2}$ are members of $\mathscr{L}(\mathscr{U}, \mathscr{V}$, there exist $B_{1}^{\prime}, B_{1}^{\prime \prime}, B_{2}^{\prime}, B_{2}^{\prime \prime}$ in $\mathscr{L}, U_{1}, U_{2}$ in $\mathscr{U}$, and $V_{1}, V_{2}$ in $\mathscr{V}$ with $B_{i}^{\prime} \subseteq U_{i} \subseteq B_{i}$ $\subseteq V_{i} \subseteq B_{i}^{\prime \prime}$ and $\left(\mu_{L}\right)_{p}\left(B_{i}^{\prime \prime} \backslash B_{i}^{\prime}\right)<\varepsilon / 2$ for $i=1$ and 2. Now if $A^{\prime}=B_{1}^{\prime} \backslash B_{2}^{\prime \prime}$ and $A^{\prime \prime}=B_{1}^{\prime \prime} \backslash B_{2}^{\prime}$, $C=U_{1}\left|V_{2}, T=V_{1}\right| U_{2}$ then $A^{\prime}, A^{\prime \prime} \in \mathscr{H}(\mathscr{L}), \quad C \in \mathscr{U} \mid \mathscr{V}=\mathscr{A}, \quad T \in \mathscr{V} \| \mathscr{U}=\mathscr{B}$ and $A^{\prime} \subseteq C \subseteq A \subseteq T \subseteq A^{\prime \prime}$ with

$$
\left(\hat{\mu}_{D}\right)_{p}\left(A^{\prime \prime} \backslash A^{\prime}\right) \leqq\left(\mu_{L}\right)_{p}\left(B_{1}^{\prime \prime} \backslash B_{1}^{\prime}\right)+\left(\mu_{L}\right)_{p}\left(B_{2}^{\prime \prime} \backslash B_{2}^{\prime}\right)<\varepsilon .
$$

COROLLARY 3.1.1. The conclusion of the preceding theorem is true if $\mu$ is a nondecreasing, real-valued modular function on $\mathscr{L}$ and in the definition of $\mathscr{L}(\mathscr{U}, \mathscr{V})$, we replace " $\left(\mu_{L}\right)_{p}\left(A^{\prime \prime} \backslash A^{\prime}\right)<\varepsilon$ " by $\mu\left(A^{\prime \prime}\right)-\mu\left(A^{\prime}\right)<\varepsilon$.

In a topological setting, we have the following:

COROLlARY 3.1.2. Let $\mathscr{L}$ be a lattice of subsets of a topological space $X$ and $\mu$ a modular function on $\mathscr{L}$ with values in the topological abelian group $\mathscr{G}$. Suppose that for each $A$ in $\mathscr{L}$, each $\varepsilon>0$, and each $p \in P$, there exist $A^{\prime}, A^{\prime \prime}$ in $\mathscr{L}$ such that $\overline{A^{\prime}} \subseteq A$ $\subseteq\left(A^{\prime \prime}\right)^{\mathrm{int}}, \overline{A^{\prime}}$ is countably compact and $\left(\mu_{L}\right)_{p}\left(A^{\prime \prime} \backslash A^{\prime}\right)<\varepsilon$. Then $\mu$ determines a unique countably additive function defined on the ring generated by $\mathscr{L}$ and with values in $\mathscr{G}$.

Proof. Let $\mathscr{U}$ be the family of closed countably compact sets and $\mathscr{V}$ be the family of open sets.

In the even more special case in which $\mathscr{L}$ is the lattice of closed compacta of a topological space, we may let $\mathscr{U}=\mathscr{L}$ and $\mathscr{V}$ be the collection of open sets and the 
conclusion of the theorem still holds. This generalizes a similar result of Kodaira [4] concerning "contents" defined on the class of all compacta of a locally compact, Hausdorff space.

\section{ChAPTER IV}

In the extension of a modular function on a lattice to a unique additive function on a semiring or the extension of an additive function on a semiring to a unique additive function on a ring, the construction of the extended function is purely algebraic. However, in the problem of extending a countably additive function $\mu$ on a ring $\mathscr{R}$ to a unique countably additive function $\hat{\mu}$ on the $\sigma$-ring $\Sigma(\mathscr{R}), \hat{\mu}$ must be constructed in terms of the topological as well as the algebraic structure of the range.

Under the assumption of a rather weak form of bounded variation, Takahashi [8] has been able to extend a topological abelian group valued countably additive function on a ring $\mathscr{R}$ to a unique countably additive function on the smallest $\delta$-ring containing $\mathscr{R}$.

We shall restrict ourselves to the special case in which the range of $\mu$ is in a Banach space $E$. If a countably additive extension $\hat{\mu}$ on $\Sigma(\mathscr{R})$ is possible, $\mu$ must be bounded (a consequence of the Uniform Boundedness Principle) so we shall assume that $\mu$ is bounded on $\mathscr{R}$. Now if $\gamma \in E^{*}$, then $\gamma \circ \mu$ has a unique countably additive scalar-valued extension $\mu_{y}$ on $\Sigma(\mathscr{R})$. For each $S$ in $\Sigma(\mathscr{R}), \hat{\mu}(S)(\gamma)=\mu_{\gamma}(S)$, considered as a function in $\gamma$, is a member of $E^{* *}$.

Thus $\hat{\mu}: \Sigma(\mathscr{R}) \rightarrow E^{* *}$ and is weak* countably additive. If the range of $\mu$ were actually in $E$, then $\hat{\mu}$ would be countably additive. Kluvanek [3] has shown that the range of $\hat{\mu}$ is a subset of $E$ if and only if the weak closure of the range of $\mu$ is weakly sequentially compact. He also showed that the following two conditions are equivalent for a countably additive function $\mu$ on a ring $\mathscr{R}$ and either suffices to ensure that the extension take values in $E$.

(i) for any nondecreasing sequence $\left\{A_{i}\right\}_{1}^{\infty}$ in $\mathscr{R},\left\{\mu\left(A_{i}\right)\right\}_{1}^{\infty}$ converges in $E$.

(ii) for any disjoint sequence $\left\{A_{i}\right\}_{1}^{\infty}$ in $\mathscr{R}, \sum_{1}^{\infty} \mu\left(A_{i}\right)$ converges in $E$.

Now (i) and (ii) are sufficient and (clearly) necessary for such an extension. We can easily show that a countably additive function $\mu$ on a semiring $\mathscr{H}$ has a unique countably additive extension $\hat{\mu}: \Sigma(\mathscr{H}) \rightarrow E$ if and only if (ii) holds. However, the relative weak sequential compactness is not sufficient nor is (i) sufficient even for nonnegative valued countably additive functions: let $\mathscr{H}=\{(n, n+1]: n$ is an integer $\}$ and let $\mu(n, n+1]=1$ then $\mu$ is countably additive, real-valued, has its range compact and satisfies (i) but does not have a real-valued extension to $\Sigma(\mathscr{H})$.

It seems likely that a modular function satisfying (i) on a lattice and having its unique ring extension countably additive would also have a countably additive extension to the $\sigma$-ring.

Were we able to obtain the sufficiency of (i) for a modular function on a lattice, we could establish the following 
CONJECTURE. Let $\mu: \mathscr{L} \rightarrow E$ be modular and have its unique ring extension countably additive. If the range of $\mu$ is relatively weakly sequentially compact, then there is a unique countably additive extension $\hat{\mu}$ on $\Sigma(\mathscr{L})$ with values in $E$.

Even though this conjecture can not be established at this date, some progress toward a lattice to $\sigma$-ring extension can be made and we consider results in that direction for the remainder of the present chapter.

The lattice to $\sigma$-ring extension problem may be broken into three main components.

(i) For each $\gamma$ in $E^{*}$, we must be able to extend $\gamma \circ \mu$ to a unique scalar-valued, countably additive function $\mu_{y}$ on $\Sigma(\mathscr{L})$. This will require that $\mu_{\gamma}$ be bounded for each $\gamma$ in $E^{*}$; this is true if and only if the restriction of $\mu_{\gamma}$ to the ring $\mathscr{R}(\mathscr{L})$ is bounded. Now if $\bar{\mu}$ is the unique ( $E$-valued) additive extension of $\mu$ to $\mathscr{R}(\mathscr{L})$, then $\gamma \circ \bar{\mu}=\mu_{\gamma}$ on $\mathscr{R}(\mathscr{L})$ so $\bar{\mu}$ is weakly bounded and, hence, bounded; this is true if and only if $\mu_{L}$ is bounded (recall that $\mu_{L}(B)=\sup \left\{\left\|\sum_{1}^{n} \mu\left(A_{i}\right)-\mu\left(C_{i}\right)\right\|:\left\{A_{i} \mid C_{i}\right\}_{1}^{n}\right.$ is pairwise disjoint and for each $i, A_{i}$ and $C_{i}$ are members of $\mathscr{L}, A_{i} \subseteq C_{i}$, and $\left.A_{i} \mid C_{i} \subseteq B\right\}$ ). To ensure countable additivity of $\mu_{y}$ we may need some type of weak (in an $E^{*}$ sense) regularity.

(ii) Considering $E$ as a subset of $E^{* *}$, we must show that there is an extension of $\mu$ to a countably additive function $\hat{\mu}$ on $\Sigma(\mathscr{L})$ but with values in $E^{* *}$. By (i), $\mu_{\gamma}$ is a countably additive scalar-valued function on $\Sigma(\mathscr{L})$ and for each $S \in \Sigma(\mathscr{L})$, $\hat{\mu}(S)=\mu_{(\cdot)}(s)$ is a function on $E^{*}$. The uniqueness of the scalar extension shows that $\hat{\mu}(S)$ is linear on $E^{*}$ and it is easily seen that $\hat{\mu}(S)$ is continuous on $E^{*}$. Thus, for each $S$ in $\Sigma(\mathscr{L}), \hat{\mu}(S) \in E^{* *}$ and $\hat{\mu}$ is easily seen to be weak* countably additive in $E^{* *}$.

(iii) We must ensure that $\hat{\mu}$ take values in $E$. One way of ensuring this is to require that values of $\hat{\mu}$ be approximated in the weak* topology in $E^{* *}$ by values of $\mu$ and require that the range of $\mu$ have its weak* closure in $E^{* *}$ be weak* compact.

For the remainder of this chapter, we shall assume that $E$ is a real Banach space and that $\mathscr{L}$ is a lattice of subsets of a set $X$ and $\phi \in \mathscr{L}$. Let $\nu: \mathscr{L} \rightarrow \boldsymbol{R}$ be modular with $\nu(\phi)=0$. In the study of lattice functionals [6, p. 4.14] variations have been defined which, in the case $\phi \in \mathscr{L}$, reduce to the following. Let $A \subseteq X$; then

$$
\begin{aligned}
& |\nu|(A)=\sup \left\{\sum_{i=2}^{n}\left|\nu\left(A_{i}\right)-\nu\left(A_{i-1}\right)\right|\right\}, \\
& \nu^{+}(A)=\sup \left\{\sum_{i=2}^{n}\left(\max \left(0, \nu\left(A_{i}\right)-\nu\left(A_{i-1}\right)\right)\right)\right\}, \\
& \nu^{-}(A)=\sup \left\{-\sum_{i=2}^{n}\left(\min \left(0, \nu\left(A_{i}\right)-\nu\left(A_{i-1}\right)\right)\right)\right\},
\end{aligned}
$$

where in each case, the supremum is taken over all finite increasing sequences $\left\{A_{i}\right\}_{1}^{n}$ of members of $\mathscr{L}$ such that $\bigcup_{1}^{n} A_{i} \subseteq A$. 
It can be shown (1) that $|\nu|, \nu^{+}, \nu^{-}$are modular, monotone increasing, extended real valued functions on $\mathscr{L}$; (2) that $|\nu|=\nu^{+}+\nu^{-}$; and (3) that if $|\nu|$ never takes on the value " $\infty$ " then $\nu(A)=\nu^{+}(A)-\nu^{-}(A)$ on $\mathscr{L}$.

Pettis [7] has proved the following

LEMMA. Let $\mathscr{C}$ be a $\delta$-lattice of closed compacta in a topological space $X$ and $\mathscr{U} a$ collection of open sets such that $\mathscr{C} \mid \mathscr{U} \subseteq \mathscr{C}$ and $\mathscr{U}|| \mathscr{C} \subseteq \mathscr{U}$. Let $\nu: \mathscr{C} \rightarrow \boldsymbol{R}$ be such that

(i) $\nu$ is monotone increasing and modular,

(ii) if $C \in \mathscr{C}$ and $\varepsilon>0$ then there exist $C^{\prime} \in \mathscr{C}, U \in \mathscr{U}$ such that $C \subseteq U \subseteq C^{\prime}$ and $\nu\left(C^{\prime}\right)-\nu(C)<\varepsilon$.

Then there is a unique, nonnegative, extended real-valued, countably additive $\hat{v}$ on the $\sigma$-ring $\Sigma(\mathscr{C})$ generated by $\mathscr{C}$, such that

$$
\hat{\nu}(S)=\sup \{\nu(C): C \in \mathscr{C}, C \subseteq S\} \quad \text { for } S \in \Sigma(\mathscr{C}) .
$$

We note that $\hat{v}$ will be real-valued if and only if $\nu_{L}$ is bounded.

We now have the following extension of Pettis' result.

THeOREM 4.1. Let $\mathscr{C}$ be a $\delta$-lattice of closed compacta of $X$ with $\phi \in \mathscr{C}$, and $\mathscr{U} a$ family of open sets with $\mathscr{C} \mid \mathscr{U} \subseteq \mathscr{C}$ and $\mathscr{U} \| \mathscr{C} \subseteq \mathscr{U}$. Let $E$ be a real Banach space and $\mu: \mathscr{C} \rightarrow E$ be such that

(i) $\mu$ is modular and $\mu(\phi)=0$,

(ii) $\mu_{L}$ is bounded on $2^{x}$,

(iii) if $C \in \mathscr{C}, \varepsilon>0$, and $\gamma \in E^{*}$, then there exist $C^{\prime} \in \mathscr{C}$ and $U \in \mathscr{U}$ such that $|\gamma \circ \mu|\left(C^{\prime} \mid C\right)<\varepsilon$ and $C \subseteq U \subseteq C^{\prime}$.

Then $\mu$ has a unique extension $\hat{\mu}$ on $\Sigma(\mathscr{C})$ to $E^{* *}$ with $\hat{\mu}$ weak* countably additive. Moreover the range of $\hat{\mu}$ is in the weak ${ }^{*}$ closure of the range of $\mu$ (considered as a subset of $\left.E^{* *}\right)$.

Proof. Let $\gamma \in E^{*}$; then $\gamma \circ \mu$ is a real-valued, modular function on $\mathscr{C}$ and $(\gamma \circ \mu)_{L}$ $\leqq\|\gamma\| \cdot \mu_{L}$ so by (ii), $(\gamma \circ \mu)_{L}$ is bounded. Now $|\gamma \circ \mu| \leqq(\gamma \circ \mu)_{L}$, so $|\gamma \circ \mu|$ is bounded and $(\gamma \circ \mu)^{+}$and $(\gamma \circ \mu)^{-}$are nonnegative real-valued. It is easily seen from (iii) that $(\gamma \circ \mu)^{+}$and $(\gamma \circ \mu)^{-}$satisfy the hypothesis of the preceding lemma and the existence of unique countably additive extensions $\mu_{\gamma}^{+}$and $\mu_{\gamma}^{-}$on $\Sigma(\mathscr{C})$ is ensured. Moreover, (ii) ensures that these extensions are real valued. Let $\mu_{\gamma}=\mu_{\gamma}^{+}-\mu_{\gamma}^{-}$, then $\mu_{\gamma}$ is the unique countably additive extension of $\gamma \circ \mu$ to $\Sigma(\mathscr{C})$ with real values. Now $\hat{\mu}(S)(\gamma)=\mu_{\gamma}(S)$, considered as a function in $\gamma$, is in $E^{* *}$ and so $\hat{\mu}: \Sigma(\mathscr{C}) \rightarrow E^{* *}$ is an extension of $\mu$ and is countably additive in the weak* topology of $E^{* *}$.

Let $\gamma_{1}, \gamma_{2}, \ldots, \gamma_{n}$ be arbitrary members of $E^{*}, S \in \Sigma(\mathscr{C})$, and let $\varepsilon>0$. For each $i(1 \leqq i \leqq n),\left(\gamma_{i} \circ \mu\right)^{+}$and $\left(\gamma_{i} \circ \mu\right)^{-}$are monotone increasing modular functions on $\mathscr{C}$ and there exist $C_{i}^{+}, C_{i}^{-} \in \mathscr{C}$ such that $C_{i}^{+} \subseteq S, C_{i}^{-} \subseteq S$ with $\left(\gamma_{i} \circ \mu\right)^{+}(S)-\left(\gamma_{i} \circ \mu\right)^{+}\left(C_{i}^{+}\right)$ $<\varepsilon / 2$ and $\left(\gamma_{i} \circ \mu\right)^{-}(S)-\left(\gamma_{i} \circ \mu\right)^{-}\left(C_{i}^{-}\right)<\varepsilon / 2$. Now if $C=\bigcup_{1}^{n} C_{i}^{+} \cup \bigcup_{1}^{n} C_{i}^{-}$, then for each $i, \mu_{\gamma_{i}}^{+}(S)-\left(\gamma_{i} \circ \mu\right)^{+}(C)<\varepsilon / 2$ and $\mu_{\gamma_{i}}^{-}(S)-\left(\gamma_{i} \circ \mu\right)^{-}(C)<\varepsilon / 2$. Thus $\mid\left(\mu_{\gamma_{i}}\right)(S)$ $-\left(\gamma_{i} \circ \mu\right)(C) \mid<\varepsilon$ and so $\left|\hat{\mu}(S)\left(\gamma_{i}\right)-\left(\gamma_{i} \circ \mu\right)(C)\right|<\varepsilon$. Hence $\hat{\mu}(S)$ is in the weak* closure of the range of $\mu$. 
COROLlaRY 4.1.1. Let $\mathscr{C}$ be a $\delta$-lattice of closed compacta of a topological space $X$ with $\phi \in \mathscr{C}$ and let $\mathscr{U}$ be a family of open sets such that $\mathscr{C} \mid \mathscr{U} \subseteq \mathscr{C}$ and $\mathscr{U} \| \mathscr{C} \subseteq \mathscr{U}$. Let $E$ be a real Banach space and $\mu: \mathscr{C} \rightarrow E$ be such that

(i) $\mu$ is modular and $\mu(\phi)=0$;

(ii) $\mu_{L}$ is bounded on $2^{x}$;

(iii) if $C \in \mathscr{C}, \varepsilon>0$ and $\gamma \in E^{*}$, then there exist $C^{\prime} \in \mathscr{C}, U \in \mathscr{U}$ such that $C \subseteq$ $U \subseteq C^{\prime}$ and $|\gamma \circ \mu|\left(C^{\prime} \mid C\right)<\varepsilon$;

(iv) the range of $\mu$ is relatively weakly sequentially compact.

Then $\mu$ has a unique countably additive extension $\hat{\mu}$ on $\Sigma(\mathscr{C})$ with values in $E$.

Proof. By Theorem $4.1, \mu$ has a unique countably additive extension $\hat{\mu}$ on $\Sigma(\mathscr{C})$ and with values in $E^{* *}$. The Eberlein-Šmulian Theorem states that if the weak closure of a set in $E$ is weakly sequentially compact in $E$, then it is weakly compact in $E$. Thus if $\mu(\mathscr{C})$ is the range of $\mu$ and $\hat{\mu}(\Sigma(\mathscr{C}))$ is the range of $\hat{\mu}$, then by the conclusion of Theorem 4.1,

$$
\hat{\mu}(\Sigma(\mathscr{C})) \subseteq \overline{\mu(\mathscr{C}})^{w k} \subseteq E .
$$

We note in the special case in which $\mathscr{C}$ is the $\delta$-lattice of all closed compacta, we need only specify (i), (ii), (iii), and (iv) to ensure an extension. Properties (i) and (ii) are also necessary for such an extension. By a result of Bartle, Dunford, and Schwartz, [1, p. 299] any countably additive function on a $\sigma$-ring has its range relatively weakly compact so (iv) is also necessary. We also note that if we require $\hat{\mu}$ to be "inner regular with respect to $\mathscr{C}$ " in a sufficiently strong sense (e.g. $\mu_{L}$ regularity) then (iii) is also necessary.

\section{BIBLIOGRAPHY}

1. R. Bartle, N. Dunford and J. Schwartz, Weak compactness and vector measures, Canad. J. Math. 7 (1955), 289-305.

2. N. Dinculeanu and I. Kluvanek, On vector measures, Proc. London Math. Soc. (3) 17 (1967), 505-512.

3. I. Kluvanek, Theory of vector-valued measures, Part II, Mat.-Fyz. Casopis Sloven. Akad. Vied 1 (1966), 76-81. (Russian)

4. K. Kodaira, Uber die Beziehung zwischen den Massen und den Topologien in einer Gruppe, Proc. Phys.-Math. Soc. Japan 23 (1941), 67-119.

5. J. von Neumann, Functional operators, Vol. I: Measures and integrals, Princeton Univ. Press, Princeton, N. J., 1950.

6. B. J. Pettis, Notes on measure theory, Part I: Lattices and lattice functionals, mimeographed notes, Tulane University, 1951.

7. - On extensions of measures, Ann. of Math. (2) 54 (1951), 186-197.

8. M. Takahashi, On topological-additive-group-valued measures, Proc. Japan Acad. 42 (1966), 330-334.

University of North Carolina, Chapel Hill, North Carolina 